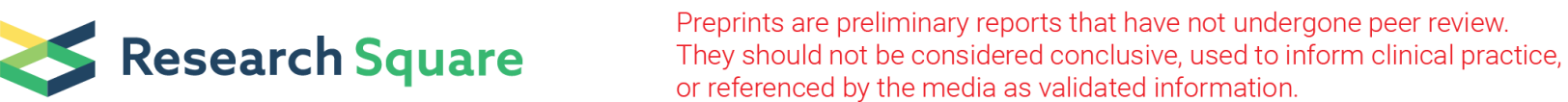

\section{In vitro and in vivo evaluation of cytotoxic effect of targeted agents alone or in combination with chemotherapy for the treatment of adenoid cystic carcinoma}

\section{Teresa Savarese}

University of Brescia: Universita degli Studi di Brescia

\section{Andrea Abate}

University of Brescia: Universita degli Studi di Brescia

Ram Manohar Basnet

University of Brescia: Universita degli Studi di Brescia Luigi Lorini

University of Brescia: Universita degli Studi di Brescia Cristina Gurizzan

University of Brescia: Universita degli Studi di Brescia

Michele Tomasoni

University of Brescia: Universita degli Studi di Brescia

Davide Lombardi

University of Brescia: Universita degli Studi di Brescia

\section{Davide Tomasini}

University of Brescia: Universita degli Studi di Brescia

\section{Daniela Zizioli}

University of Brescia: Universita degli Studi di Brescia

\section{Maurizio Memo}

University of Brescia: Universita degli Studi di Brescia

\section{Alfredo Berruti}

University of Brescia: Universita degli Studi di Brescia

Sara Anna Bonini ( $\nabla$ sara.bonini@unibs.it)

University of Brescia: Universita degli Studi di Brescia

\section{Sandra Sigala}

University of Brescia: Universita degli Studi di Brescia

\section{Paolo Bossi}

University of Brescia: Universita degli Studi di Brescia 


\section{Research Article}

Keywords: adenoid cystic carcinoma, ACC cell line, zebrafish embryos, tumor xenograft, standard chemotherapy, targeted therapy

Posted Date: April 27th, 2021

DOl: https://doi.org/10.21203/rs.3.rs-446009/v1

License: (c) (i) This work is licensed under a Creative Commons Attribution 4.0 International License.

Read Full License 


\section{Abstract \\ Purpose}

Adenoid cystic carcinoma (ACC) is a rare malignancy characterized by high incidence of relapse. When relapsing, ACC has an indolent but relentless behaviour, thus leading to a poor long-term prognosis. The treatment of choice of relapsing ACC remains surgery followed by radiotherapy, whenever feasible. Therapeutic weapons are limited to systemic drugs. The most widely used chemotherapy regimen is the combination of cisplatin and doxorubicin, however with low response rate and not long lasting; there is also a lack of alternatives for second line therapies in case of disease progression. Therefore, a more comprehensive strategy aimed at identifying at preclinical level the most promising drugs or combination is clearly needed.

\section{Methods}

In this study, the cytotoxic effects of two standard chemotherapy drugs, cisplatin and doxorubicin, and of five targeted therapy-drugs was tested in vitro, on an h-TERT immortalized ACC cell line. The same drugs were also tested in vivo, on zebrafish embryos with ACC tumoral cell xenograft. Then, combinations of one standard chemotherapy drug plus one targeted therapy drug were also evaluated, in order to find the best treatment strategy for ACC.

\section{Results}

Data obtained demonstrated that both vorinostat and olaparib significantly increased the standard chemotherapy cytotoxic effects, suggesting new interesting therapeutic options for ACC.

\section{Conclusion}

Data obtained in the present study provide valid new therapeutic strategies for ACC to be translated in a prospective clinical trial.

\section{Introduction}

Adenoid cystic carcinoma (ACC) is a rare malignancy arising mostly from salivary glands and from other sites, as trachea, bronchi, breast and skin, with an incidence of 4.5 cases per 100,000 individuals (Ellington et al. 2012). It represents $20 \%$ of malignant tumors of the major salivary glands and $58 \%$ of the minor salivary glands (Coca-Pelaz et al. 2015). ACC is reported to relapse in till 50-60\% of the cases, both locally and at distant sites (Xu et al. 2017; Terhaard et al. 2004); mainly metastatic sites are represented by lung (70\% of cases), followed by bone (6\%), liver (3\%) and rarely brain or other sites (Xu et al. 2018). 
Generally, when relapsing, ACC has an indolent but relentless behaviour, thus leading to a poor long-term prognosis; 10 -year OS ranges from $52 \%$ to $65 \%$ in several retrospective series (Ciccolallo et al. 2009; van Weert et al. 2013).

Locoregional or distant recurrences represent a crucial part of the patient journey, as negatively impacting on prognosis and with higher risk of patient's quality of life deterioration. The treatment of choice of relapsing ACC remains surgery followed by radiotherapy, whenever feasible. In case of absence of indications for locoregional treatments, with a patient experiencing symptomatic disease or at high risk of complications, therapeutic weapons are limited to systemic drugs.

The most widely used chemotherapy regimen is the combination of cisplatin and doxorubicin, however with response rate (RR) of $25 \%$ and not long lasting; there is also a lack of alternatives for second line therapies in case of disease progression (Laurie et al. 2011). Several target agents have been studied in ACC, mostly as single agents and in the absence of molecular selection. Recently, the most promising results have been obtained with multikinase inhibitor lenvatinib and with antiangiogenetic drugs as sorafenib and axitinib; however, these drugs reported a response rate of about $15 \%$ with a non-negligible burden of adverse events (Thomson et al. 2015; Keam et al. 2020; Tchekmedyian et al. 2019; Locati et al. 2020). The most frequently reported molecular alteration, aside the MYB/MYB1 pathognomonic fusions, is the NOTCH-1 mutation (Ho et al. 2019); in this regard, tailored targeted approaches have been studied and are currently ongoing in NOTCH-mutated ACC (Ferrarotto et al. 2020). Overall, targeted agents seem to benefit a small quote of recurrent and/or metastatic (RM) ACC, at the price of drug-induced toxicities. Therefore, a more comprehensive strategy aimed at identifying at preclinical level the most promising drugs or combination is clearly needed.

The primary aim of our study is the evaluation in vitro and in vivo of new therapeutic strategies that can expand therapeutic possibilities available for patients with RM ACC.

\section{Materials And Methods}

\section{Cell culture}

The human ACC (hTERT) cell line, derived from a primary untreated and predominantly cribriform ACC of the tongue base immortalized using h-TERT transfection, was kindly given by Prof. Adel El-Naggar (Department of Pathology, The University of Texas, MD Anderson Cancer Center, Houston, TX, U.S.A.). Cells were maintained in a medium containing 82\% RPMI-1640 (Sigma-Aldrich , St. Louis, Missouri, USA), 15\% fetal bovine serum (FBS, Life Technologies, Carlsbad, California, USA), 1\% penicillin/streptomycin (Life Technologies), 2\% L-glutamine (Life Technologies), as previously reported (Li et al. 2014). ACC (hTERT) cells doubling time at $37^{\circ} \mathrm{C}$ and $5 \% \mathrm{CO}_{2}$ was calculated according to ATCC (American Type Culture Collection, Virginia, USA) indication, with the following formula: $D T=T \ln 2 / \ln \left(X_{e} / X_{b}\right)$, where $T$ is the incubation time in any units, $X_{b}$ is the cell number at the beginning of the incubation time, $X_{e}$ is the cell number at the end of the incubation time. The same protocol was applied to calculate the doubling 
time at $32^{\circ} \mathrm{C}$. Cells were authenticated by the GenePrint 10 System (Promega Italia, Milan, Italy), according to the protocols suggested by the manufacturer. Cells were used between passage 144 and 155 and periodically tested for mycoplasma.

\section{Cells treatment}

ACC (hTERT) cells ( 15.000 cells'well) were plated in 24-well plates with complete medium. Preliminary experiments were conducted to set up the optimal cell number and the range of drug concentrations used in the subsequent experiments. For the concentration-response curves, cells were exposed to the following drugs concentration: cisplatin (Selleckchem Chemicals, Houston, Texas, USA) (0.03-24 $\mu \mathrm{M}$ ); doxorubicin (Selleckchem Chemicals) (0.001-1 $\mu \mathrm{M}$ ); lenvatinib (MedChem Express, Monmouth Junction, New Jersey, USA) (0.05-2 $\mu \mathrm{M})$; vorinostat (MedChem Express) (0.1-9 $\mu \mathrm{M})$; everolimus (Selleckchem Chemicals) (0.5-250 nM); palbociclib (Selleckchem Chemicals) (0.03-9 uM); olaparib (Selleckchem Chemicals) (0.15-18 $\mu \mathrm{M})$. According to cells doubling time ( 50 hours), ACC (hTERT) cells were treated with the drugs for 4 days.

\section{Cell viability and cell proliferation Assay}

Cell viability was assessed by 3-(4,5-Dimethyl-2-thiazol)-2,5-diphenyl-2H-tetrazolium bromide (MTT) dye reduction assay according to the manufacturer protocol (Sigma Aldrich) and performed as described in Abate et al. (2020). Cell proliferation rate was evaluated with TC20 automated cell counter (Bio-Rad, Segrate, Milan, Italy). Briefly, vehicle- and drugs-treated cells were trypsinized and suspended in culture medium followed by trypan blue dilution (1:2). The parameter settings were established according to the manufacturer instructions. Ten microliters of the mixture were loaded into the opening of the TC20 counting slide. The gating was selected as optimal, based on preliminary validation studies performed.

\section{Drug combination experiments}

Drug combination experiments were performed to evaluate drug interactions on ACC cell viability, according to the Chou and Talaly method (Chou and Talalay 1984). Cells were treated for 4 days with the following drugs, used alone or in combination: cisplatin $(0.018-13.2 \mu \mathrm{M})$ and palbociclib $(0.020-$ $14.742 \mu \mathrm{M})$, doxorubicin $(0.002-0.255 \mu \mathrm{M})$ and palbociclib $(0.026-3 \mu \mathrm{M})$, cisplatin $(0.187-21.3 \mu \mathrm{M})$ and vorinostat $(0.064-7.26 \mu \mathrm{M})$, doxorubicin $(0.002-0.255 \mu \mathrm{M})$ and vorinostat $(0.14-16 \mu \mathrm{M})$, cisplatin $(0.1-30.37 \mu \mathrm{M})$ and everolimus $(0.41-157.46 \mathrm{nM})$, doxorubicin $(0.002-0.255 \mu \mathrm{M})$ and everolimus $(0.62-70.3 \mathrm{nM})$, cisplatin $(0.75-25.66 \mu \mathrm{M})$ and olaparib $(0.874-29.74 \mu \mathrm{M})$, doxorubicin $(0.002-0.255 \mu \mathrm{M})$ and olaparib $(0.23-26.6 \mu \mathrm{M})$, vorinostat $(0.018-13.5 \mu \mathrm{M})$ and palbociclib $(0.02-$ $14.58 \mu \mathrm{M})$. Drug concentrations used in the combinations followed a fixed dilution ratio, as recommended for the most efficient data analysis (Chou 2006). Data were analysed using the CompuSyn software (ComboSyn inc. Paramus, NJ, USA) as described in Abate et al. (2020).

\section{Collection of Zebrafish eggs and fish maintenance}


All zebrafish were handled according to national and international guidelines (EU Directive 2010/63/EU), following protocols approved by the local committee (OPBA protocol no. 211B5.24) and authorized by the Ministry of Health (authorization no. 393/2017-PR). Healthy adult wild-type zebrafish (AB strain) were used for egg production. Fishes were maintained under standard laboratory conditions (Westerfield 2000 ), at $28^{\circ} \mathrm{C}$ on a constant 14 -hour light/10-hour dark cycle. Fish were fed thrice daily with a combination of granular dry food and fresh artemia (Special Diet Services, SDS Diets; LBS Biotech, Horley, UK). Collection of zebrafish eggs and maintenance of developing embryos has been performed as previously described (Gianoncelli et al. 2019).

\section{Zebrafish single-drug treatment concentration}

Embryos maintained in fish water plus PTU were manually dechorionated at 48 hours post-fertilization (hpf) and treated for 3 days with each single drug at these concentration range: cisplatin $(25-50 \mu \mathrm{M})$,

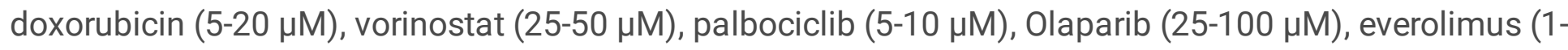
$5 \mu \mathrm{M})$. For each drug the toxicity was tested, and the final effective concentration was set for subsequent experiments.

\section{Tumor Xenograft}

ACC cells $\left(1.25 \times 10^{6}\right.$ cells) were labelled with the vital red fluorescent dye CellTrackerTM CM-Dil (final concentration $0.66 \mathrm{ng} / \mathrm{mL}$; Thermo Fisher Scientific, Milan, Italy), and resuspended in $25 \mu \mathrm{L}$ of PBS for microinjections. Tumor xenograft was performed as previously described (Gianoncelli et al. 2019). Drugs or solvents were directly added to the PTU-fish water and after 3 days of treatment (T3), pictures were taken. The tumour areas of vehicle- and drug-treated groups at T0 and T3 were analysed with Axio Zoom Fluorescent Microscope (Zeiss) and measured with Noldus DanioScopeTM software (Noldus information Technology). Data obtained were analysed by GraphPad Prism software version 6.01.

\section{Statistical analysis}

Data analysis was performed using GraphPad Prism software version 5.02 (GraphPad Software, La Jolla, $\mathrm{CA}$ ). Statistical analysis was carried out using one-way ANOVA analysis with a post hoc test (Bonferroni's test) for multiple comparisons, considering $p<0.05$ as threshold for statistical significance. $I_{50}$ values for each drug were calculated by non-linear regression of the concentration-response curves. Data are expressed as mean \pm SEM of three independent experiments, unless otherwise specified. Cytotoxicity experiments were carried out at least three times, each point run in triplicate.

\section{Results}

\section{Standard chemotherapy-and targeted therapy-drugs induced cytotoxicity in ACC cell line}

ACC (hTERT) cell line doubling time was firstly evaluated (Figure S1). Being the cells doubling time around 50 hours, ACC cells were treated with the drugs for 4 days. 
The cytotoxicity of the two standard chemotherapeutic agents (cisplatin and doxorubicin) and five targeted therapy-drugs (namely, everolimus, palbociclib, olaparib, vorinostat, and lenvatinib) were tested on ACC (hTERT) cell line.

Exposure of ACC (hTERT) cells to increasing concentrations of cisplatin (0.03-24 $\mu \mathrm{M})$ and doxorubicin (0.001-1 $\mu \mathrm{M})$ for 4 days led to a concentration-dependent reduction of cell viability, analysed by MTT assay. Sigmoidal concentration-response function was applied to calculate the $\mathrm{IC}_{50}$ value, that was, respectively, $3 \mu \mathrm{M}$ (95\% confidence interval $(\mathrm{Cl}): 2.3-3.5 \mu \mathrm{M})$ and $0.013 \mu \mathrm{M}(95 \% \mathrm{Cl}: 0.01-0.017 \mu \mathrm{M})$ for cisplatin and doxorubicin (Figure 1A-C). The standard chemotherapy drugs were highly active in inducing cytotoxicity, as their efficacy reached over the $90 \%$ at the highest concentration tested.

Also, a cell count was performed in order to evaluate the effect on the cell proliferation rate. As reported in Figure 1B-D, cell count confirmed data obtained in the MTT viability test.

We then evaluated the effect of ACC potentially effective target-therapy drugs. Among the drugs tested, the four drugs listed below resulted to be effective in reducing cell viability, considering drug concentrations compatible with clinical practice: vorinostat (0.1-9 $\mu \mathrm{M})$, olaparib (0.15-18 $\mu \mathrm{M})$, palbociclib (0.03-9 $\mu \mathrm{M})$, and everolimus $(0.5-250 \mathrm{nM})$ (Figure 2A-C-E-G). The $\mathrm{IC}_{50}$ values were derived from the concentration-response functions: vorinostat $1.3 \mu \mathrm{M}(95 \% \mathrm{Cl}: 1.1-1.6 \mu \mathrm{M})$, olaparib $5.5 \mu \mathrm{M}(95 \% \mathrm{Cl}$ : 3.88.1 $\mu \mathrm{M}$ ), palbociclib $0.13 \mu \mathrm{M}$ ( $95 \% \mathrm{Cl}$ : 0.09-0.2 $\mu \mathrm{M}$ ), everolimus $0.5 \mathrm{nM}$ (95\% Cl: 0.3-0.8 nM). Drug effects on cell proliferation were also evaluated by means of cell counts, as reported in Figure 2B-D-F-H. Detailed data on drugs potency and efficacy are reported in Table 1. Lenvatinib was as well tested for cytotoxic effects on ACC (hTERT) cells, but its efficacy was very poor, remaining far above the $50 \%$ value of cell viability even at high drug concentrations (Figure S2).

\section{In vivo effects of both standard chemotherapy and target therapy drugs}

The cytotoxic effects observed in vitro was then tested in vivo in the experimental model of cell xenograft on zebrafish embryos. As zebrafish embryos were maintained at the temperature of $32^{\circ} \mathrm{C}$, ACC cell behavior was studied in terms of viability and doubling time at $32^{\circ} \mathrm{C}$ and results indicated that this cell line was able to growth and duplicate at this temperature (Figure S3). The ACC cells were injected in zebrafish embryos and the tumor area was evaluated at different times. In particular, each single drug or solvent were directly added to the fish water and after 3 days of treatment the tumor areas of vehicle- and drug-treated groups at T0 ( 2 hours after treatment) and T3 (3 days of treatment) were measured. ACC tumor area was acquired at the fluorescent microscope and analysed with the DanioScope software (Figure 3B).

As reported in Figure 3A, ACC tumor grew very fast in the injected area of the yolk sac, approximately doubling its area in 72 hours (mean area $\left(\mathrm{mm}^{2}\right) \pm$ SEM: $29.11 \pm 0.9 \times 10^{3} \mathrm{~mm}^{2}$ and $52.6 \pm 4.9 \times 10^{3} \mathrm{~mm}^{2}$ at T0 and T3, respectively). All the drugs tested significantly reduced the tumor area compared to T3vehicle. In particular, doxorubicin, vorinostat and palbociclib exposure reduced the tumor area at levels comparable to the T0 group (mean area $\left(\mathrm{mm}^{2}\right) \pm$ SEM: $24.7 \pm 1.6 \times 10^{3} \mathrm{~mm}^{2} ; 23.0 \pm 2.3 \times 10^{3} \mathrm{~mm}^{2}$ and 
$24.0 \pm 2.0 \times 10^{3} \mathrm{~mm}^{2}$, respectively). Cisplatin, everolimus and olaparib treatment, although less effective in reducing tumor area compared to the other drugs tested, led to a significant difference as compared to the T3-vehicle group (mean area $\left(\mathrm{mm}^{2}\right) \pm$ SEM: $31.4 \pm 3.4 \times 10^{3} \mathrm{~mm}^{2} ; 34.7 \pm 2.4 \times 10^{3} \mathrm{~mm}^{2}$ and $31.8 \pm$ $1.7 \times 10^{3} \mathrm{~mm}^{2}$, respectively).

\section{Vorinostat and olaparib enhanced cytotoxicity induced by standard chemotherapy drugs}

In order to evaluate the effect on ACC cells of targeted drugs combined with either doxorubicin or cisplatin, the Chou-Talalay method was applied, as described in Methods. The most promising results were obtained with the combination vorinostat plus cisplatin and vorinostat plus doxorubicin. In these cases, the combined treatments in ACC cells induced synergistic cytotoxic effects compared to each single compound. The concentration-response curves of single standard chemotherapy drug and of the combined treatments are reported in Figure 4A-B. An increase in the potency was also observed in both combinations. Results obtained were converted to $F_{a}$ values and analyzed with CompuSyn software; the combination index plots and the synergic effects are shown in Figure 4C-D. The combination index values were $<1$ starting from $0.68 \mathrm{mM}$ and $2 \mathrm{mM}$ for vorinostat and cisplatin, respectively. In the case of vorinostat/doxorubicin, combination index $<1$ was observed with $0.68 \mathrm{mM}$ vorinostat and $0.011 \mathrm{mM}$ doxorubicin.

Interestingly, olaparib treatment in ACC cells enhanced cytotoxicity induced by standard chemotherapy drugs. Indeed, both olaparib/cisplatin and olaparib/doxorubicin combined treatment increased cytotoxic effect as compared to each single-drug treatment which resulted in an increase in the potency, as reported in concentration-response curves (Figure 5A-B). The Chou-Thalalay analysis confirmed the additive effect of the combined treatments (combination index $=1$, see Figure $5 C-D$ ).

All target therapy drugs were tested in combination with standard chemotherapy drugs, but as emerged in Chou-Thalalay analysis (Figure 6), palbociclib and everolimus did not produce any significant increase in the single standard chemotherapy treatments. Specific concentration-response curves are reported in Figures4.

\section{Discussion}

Chemotherapy has been for years the mainstay of treatment of relapsed ACC not eligible to locoregional treatment, but with no proofs of benefit in increasing survival (Licitra et al. 1991, 1996; Airoldi et al. 2001). No treatment options are present up to now for second line therapy (Alfieri et al. 2017). Targeted therapy has emerged as a treatment option, but data till now available are quite disappointing. Single agents cetuximab, imatinib, dovitinib, bortezomib, cabozantinib, and bortezomib showed limited response rate (0-6\%) (Alfieri et al. 2017). Even combination therapy of chemotherapy plus imatinib or bortezomib did not show synergistic effects in unselected population (Argiris et al. 2011; Ghosal et al. 2011). These unsatisfactory results underline the importance of comprehensively dissecting the therapeutic pathways involved in ACC and widely testing in preclinical models the activity of drug(s). The burden of mutations 
in ACC is low, with a few alterations recognized as therapeutically actionable (Ho et al. 2019). This is mirrored by the paucity of trials aimed at delivering targeted treatments associated with known mutations or with altered molecular pathways. Moreover, the additive or synergistic effect of chemotherapy and targeted agents have not been widely studied at preclinical level.

In the present study, a human ACC cell line immortalized using h-TERT transfection, was used to test in vitro the pharmacological effects of standard chemotherapy and targeted therapy agents used in monotherapy or in combination, to give the preclinical bases for a pharmacological strategy for ACC treatment. We selected the most employed chemotherapy agents in ACC, cisplatin and doxorubicin (Ghosal et al. 2011), studied to verify their cytotoxic and antiproliferative effect in the ACC experimental model used, and five targeted therapy agents, vorinostat, olaparib, palbociclib, everolimus and lenvatinib. The drug concentrations used in the study are consistent with the doses actually used in the clinic and with the resulted plasmatic concentrations (Vorinostat, Olaparib, Palbociclib, Everolimus, IBM, Micromedex, DRUGDEX; van Tilburg et al. 2019; Tamura et al. 2016; Ikeda et al. 2016; Gupta et al. 2016).

The rationale underlying the targeted therapy drugs chosen to evaluate their cytotoxic activity lies on data in literature, showing that the targets of these drugs were involved in the ACC carcinogenesis and progression. Indeed, it has been shown that recurring mutations in chromatin remodelling regulations genes are involved in ACC (Frierson et al. 2013). Vorinostat, a histone deacetylase inhibitor, showed response just in $7 \%$ of the case as monotherapy, but exerted its synergistic effect with cisplatin in preclinical models (Goncalves et al. 2017; Almeida et al. 2017). Moreover, vorinostat in association with cisplatin demonstrated efficacy in depleting cancer stem cells and reducing tumor viability in ACC primary cells (Almeida et al. 2017). The PARP inhibitor olaparib was chosen because of the demonstrated presence in ACC of molecular alterations involved in DNA repair pathways, and thus possibly having a role both as single agent and in combination with chemotherapy (Ho et al. 2019). Role of cycline dependent kinase (CDK) inhibitors has been recently evaluated in an in vitro study in ACC, with evidence of synergistic antitumoral activity in combination with cisplatin (Xu et al. 2020). Everolimus may play a role in ACC, as, in an ACC cell line, Younes et al. (2006) showed the role of phosphatidylinositol 3-kinase (PI3K)-Akt-mammalian target of rapamycin (mTOR) as potentially target of therapy. Everolimus in a phase 2 study (Kim et al. 2014) showed a median PFS of 11.2 months and about $80 \%$ of stable disease, however no partial or complete response were achieved. Finally, lenvatinib is a second-generation multiple kinase inhibitor with a strong anti-angiogenic effect; it reported responses in the range of $12-16 \%$ and high rate of disease stabilization (about $70 \%$ of patients), but with half of the patients reporting toxicity of grade 3 or greater (Tchekmedyian et al. 2019; Locati et al. 2020).

Our results demonstrated the in vitro cytotoxic activity of the standard chemotherapy drugs. Among targeted agents, vorinostat reported the highest efficacy, with a cell mortality of over $90 \%$, while modest efficacy emerged for olaparib and palbociclib and poor efficacy for everolimus. Finally, lenvatinib did not induce significant cytotoxic effects in ACC (hTERT) cell line, and this poor performance could be explained by the lack of vascular components of in vitro models, that are indeed one of the main mechanisms of action of this drug. The in vitro cytotoxic effect of all the drugs tested was confirmed and 
strengthen by results obtained in the in vivo model of ACC (hTERT) cell line xenograft in zebrafish embryos. This animal model offers a valid and useful tool for in vivo first drug screening, due to the limited number of tumoral cells needed, the costs and time reduction (Zhang et al. 2015; Gianoncelli et al. 2019).

In the context of ACC tumor, several studies confirmed that a two-drug polychemotherapy gives better response rate compared with monotherapy (Lorini et al. 2021), even if with limited duration and uncertain benefit in overall survival. Therefore, the association of chemotherapy and targeted agents is a logical step to be carried out (Chabner and Roberts 2005) and we tried to demonstrate the activity of these combinations in the in vitro model of ACC cell line. Our results demonstrated that the two standard chemotherapy agents (cisplatin or doxorubicin) plus vorinostat or olaparib were effective. Indeed, vorinostat significantly increased both the efficacy and the potency of the standard chemotherapy agents and the combination exerted a synergic effect. Also olaparib improved the performance of the two chemotherapy agents, resulting in an addictive effect. These effects found their rationale in the specific molecular mechanism of the drugs: both cisplatin and doxorubicin hamper DNA replication and RNA transcription, the first inducing the formation of intra- and inter-strand cross-link of nuclear DNA (Wang and Lippard 2005), the second interacting with DNA by intercalation and inhibiting macromolecular biosynthesis. This effect finally leads to cell cycle arrest and apoptosis. Vorinostat increases the formation of hyperacetylated histones, which therefore blocks the interaction of histone with DNA and leaves it more accessible by cisplatin or doxorubicin. Therefore, the synergistic effect induced by vorinostat may be due to the action upstream of the chemotherapy agents, which facilitates and greatly enhances their effects and this result in ACC cells is in line with the finding that vorinostat sensitizes ACC tumor cells to chemotherapy (Almeida et al. 2017). Olaparib, on the other hand, preventing the repair of the DNA damages induced by platinum compounds or doxorubicin (Zhu et al. 2010), acts downstream of cisplatin and doxorubicin, adding a further barrier to cell proliferation.

Taken together, our results indicate that combined treatment with vorinostat or olaparib with standard chemotherapy agents cisplatin and doxorubicin is significantly more effective than monotherapy. These data set the basis for further studies in a dedicated prospective clinical trial.

We acknowledge that a major limitation of this study lies in the experimental model, as we could perform experiments just in a single cell line; however, up to now, the AdCC-hTERT cells are the only cell models available. Also, we did not explore the role of immunotherapy, as in this preclinical cell line model, the absence of tumor microenvironment prevented the possibility to study the interaction with the immune system. In conclusion, we demonstrated the feasibility of preclinical analyses on cell lines for the combination of chemotherapy and targeted agents in ACC, as a model to study combinations and to exploit the synergy between drugs.

\section{Declarations}

Funding: This work was supported by private donation and by local grant of the University of Brescia. 
Conflicts of interest/Competing interests: Paolo Bossi: Advisory board or conference honoraria: Merck, Sanofi, Merck Sharp \& Dohme, Sun Pharma, Angelini, Molteni, Bristol-Myers Squibb, Helsinn, GSK.

All other authors declare that they have no conflicts of interest in the area of adenoid cystic carcinoma.

Availability of data and material: All data generated or analysed during this study are included in this published article [and its supplementary information files]. Further details are available on request from the corresponding author.

Code availability: not applicable

Author's Contribution: T.S. and A.A. contributed to the collection of data, preparation of the figures and Table 1, and writing of the manuscript. R.M.B. contributed to the collection of data on zebrafish embryos and preparation of the Figure 3. L.L., C.G., M.T., D.L., D.T., D.Z., M.M., A.B. reviewed the manuscript. S.A.B. contributed to preparation of the figures, writing and establishment of the structure of this manuscript. S.S. and P.B. designed the study, contributed to the establishment of the structure of this manuscript, and acted as main supervisors.

Ethics approval: The study was conducted according to national and international guidelines (EU Directive 2010/63/EU), following protocols approved by the local committee (OPBA protocol no. 211B5.24) and authorized by the Ministry of Health (authorization no. 393/2017-PR).

Consent to participate: not applicable

Consent for publication: not applicable

\section{References}

1. Abate A, Rossini E, Bonini SA, Fragni M, Cosentini D, Tiberio GAM, et al (2020) Cytotoxic Effect of Trabectedin In Human Adrenocortical Carcinoma Cell Lines and Primary Cells. Cancers (Basel) 12:928. http://doi:10.3390/cancers12040928

2. Airoldi M, Pedani F, Succo G, Gabriele AM, Ragona R, Marchionatti S, Bumma C (2001) Phase II randomized trial comparing vinorelbine versus vinorelbine plus cisplatin in patients with recurrent salivary gland malignancies. Cancer 91:541-547. http://doi:10.1002/1097-

0142(20010201)91:3<541::aid-cncr1032>3.0.c0;2-y

3. Alfieri S, Granata R, Bergamini C, Resteghini C, Bossi P, Licitra LF, Locati LD (2017) Systemic therapy in metastatic salivary gland carcinomas: A pathology-driven paradigm?. Oral Oncol 66:58-63. http://doi:10.1016/j.oraloncology.2016.12.016

4. Almeida LO, Guimarães DM, Martins MD, Martins MAT, Warner KA, Nör JE, Castilho RM, Squarize CH (2017) Unlocking the chromatin of adenoid cystic carcinomas using HDAC inhibitors sensitize cancer stem cells to cisplatin and induces tumor senescence. Stem Cell Res 21:94-105. http://doi:10.1016/j.scr.2017.04.003 
5. Argiris A, Ghebremichael M, Burtness B, Axelrod RS, Deconti RC, Forastiere AA (2011) A phase 2 trial of bortezomib followed by the addition of doxorubicin at progression in patients with recurrent or metastatic adenoid cystic carcinoma of the head and neck: a trial of the Eastern Cooperative Oncology Group (E1303). Cancer 117:3374-3382. http://doi:10.1002/cncr.25852

6. Chabner BA, Roberts TG Jr (2005) Timeline: Chemotherapy and the war on cancer. Nat Rev Cancer 5:65-72. http://doi:10.1038/nrc1529

7. Chou TC, Talalay P (1984) Quantitative analysis of dose-effect relationships: the combined effects of multiple drugs or enzyme inhibitors. Adv Enzym Regul 22:27-55. http://doi:10.1016/00652571(84)90007-4

8. Chou TC (2006) Theoretical basis, experimental design, and computerized simulation of synergism and antagonism in drug combination studies. Pharmacol Rev 58:621-681. http://doi:10.1124/pr.58.3.10

9. Ciccolallo L, Licitra L, Cantú G, Gatta G, EUROCARE Working Group (2009) Survival from salivary glands adenoid cystic carcinoma in European populations. Oral Oncol 45:669-674. http://doi:10.1016/j.oraloncology.2008.10.010

10. Coca-Pelaz A, Rodrigo JP, Bradley PJ, Vander Poorten V, Triantafyllou A, Hunt JL, et al (2015) Adenoid cystic carcinoma of the head and neck--An update. Oral Oncol 51:652-661. http://doi:10.1016/j.oraloncology.2015.04.005

11. Ellington CL, Goodman M, Kono SA, Grist W, Wadsworth T, Chen AY, et al (2012) Adenoid cystic carcinoma of the head and neck: Incidence and survival trends based on 1973-2007 Surveillance, Epidemiology, and End Results data. Cancer 118:4444-4451. Erratum in: Cancer 2012 Nov 1; 118: 5448-5449. http:// doi:10.1002/cncr.27408

12. Everolimus. IBM Micromedex® DRUGDEX® (electronic version). IBM Watson Health, Greenwood Village, Colorado, USA. https://www.micromedexsolutions.com/. Accessed 16 January 2021

13. Ferrarotto R, Wirth LJ, Muzaffar J, Rodriguez CP, Xia B, Perez CA, et al (2020) ACCURACY a phase II trial of AL101, a selective gamma secretase inhibitor, in subjects with recurrent/metastatic (R/M) adenoid cystic carcinoma (ACC) harboring Notch activating mutations (Notchmut). Annals of Oncology 31 (suppl_4):S599-S628

14. Frierson HF Jr, Moskaluk CA (2013) Mutation signature of adenoid cystic carcinoma: evidence for transcriptional and epigenetic reprogramming. J Clin Invest 123:2783-2785. http://doi:10.1172/JCl69070

15. Ghosal N, Mais K, Shenjere P, Julyan P, Hastings D, Ward T, et al (2011) Phase Il study of cisplatin and imatinib in advanced salivary adenoid cystic carcinoma. Br J Oral Maxillofac Surg 49:510-515. http://doi:10.1016/j.bjoms.2010.09.013

16. Gianoncelli A, Guarienti M, Fragni M, Bertuzzi M, Rossini E, Abate A, et al (2019) Adrenocortical Carcinoma Xenograft in Zebrafish Embryos as a Model To Study the In Vivo Cytotoxicity of Abiraterone Acetate. Endocrinology 160:2620-2629. http://doi:10.1210/en.2019-00152 
17. Goncalves PH, Heilbrun LK, Barrett MT, Kummar S, Hansen AR, Siu LL, et al (2017) A phase 2 study of vorinostat in locally advanced, recurrent, or metastatic adenoid cystic carcinoma. Oncotarget 8:32918-32929. http://doi:10.18632/oncotarget.16464

18. Gupta A, Jarzab B, Capdevila J, Shumaker R, Hussein Z (2016) Population pharmacokinetic analysis of lenvatinib in healthy subjects and patients with cancer. Br J Clin Pharmacol 81:1124-1133. http://doi:10.1111/bcp.12907

19. Ho AS, Ochoa A, Jayakumaran G, Zehir A, Valero Mayor C, Tepe J, et al (2019) Genetic hallmarks of recurrent/metastatic adenoid cystic carcinoma. J Clin Invest 129:4276-4289. http://doi:10.1172/JCl128227

20. Ikeda M, Okusaka T, Mitsunaga S, Ueno H, Tamai T, Suzuki T, et al (2016) Safety and Pharmacokinetics of Lenvatinib in Patients with Advanced Hepatocellular Carcinoma. Clin Cancer Res 22:1385-1394. http://doi:10.1158/1078-0432.CCR-15-1354

21. Keam B, Kang EJ, Ahn M, Ock C, Lee KW, Kwon JH, et al (2020) Randomized phase II study of axitinib versus observation in patients with recurred or metastatic adenoid cystic carcinoma. J Clin Oncol 38:6503-6503. http://doi:10.1200/JC0.2020.38.15_suppl.6503

22. Kim DW, Oh DY, Shin SH, Kang JH, Cho BC, Chung JS, et al (2014) A multicenter phase II study of everolimus in patients with progressive unresectable adenoid cystic carcinoma. BMC Cancer 14:795. http://doi:10.1186/1471-2407-14-795

23. Laurie SA, Ho AL, Fury MG, Sherman E, Pfister DG (2011) Systemic therapy in the management of metastatic or locally recurrent adenoid cystic carcinoma of the salivary glands: a systematic review. Lancet Oncol 12:815-824. http://doi:10.1016/S1470-2045(10)70245-X

24. Li J, Perlaky L, Rao P, Weber RS, El-Naggar AK (2014) Development and characterization of salivary adenoid cystic carcinoma cell line. Oral Oncol 50:991-999. http://doi:10.1016/j.oraloncology.2014.06.012

25. Licitra L, Cavina R, Grandi C, Palma SD, Guzzo M, Demicheli R, Molinari R (1996) Cisplatin, doxorubicin and cyclophosphamide in advanced salivary gland carcinoma. A phase II trial of 22 patients. Ann Oncol 7:640-642. http://doi:10.1093/oxfordjournals.annonc.a010684

26. Licitra L, Marchini S, Spinazzè S, Rossi A, Rocca A, Grandi C, Molinari R (1991) Cisplatin in advanced salivary gland carcinoma. A phase II study of 25 patients. Cancer 68:1874-1877. http://doi:10.1002/1097-0142(19911101)68:9<1874::aid-cncr2820680904>3.0.co;2-s

27. Locati LD, Galbiati D, Calareso G, Alfieri S, Singer S, Cavalieri S, et al (2020) Patients with adenoid cystic carcinomas of the salivary glands treated with lenvatinib: Activity and quality of life. Cancer 126:1888-1894. http://doi:10.1002/cncr.32754

28. Lorini L, Ardighieri L, Bozzola A, Romani C, Bignotti E, Buglione M, et al (2021) Prognosis and management of recurrent and/or metastatic head and neck adenoid cystic carcinoma. Oral Oncol 115:105213. http://doi:10.1016/j.oraloncology.2021.105213

29. Olaparib. IBM Micromedex® DRUGDEX® (electronic version). IBM Watson Health, Greenwood Village, Colorado, USA. https://www.micromedexsolutions.com/. Accessed 16 January 2021 
30. Palbociclib. IBM Micromedex® DRUGDEX® (electronic version). IBM Watson Health, Greenwood Village, Colorado, USA. https://www.micromedexsolutions.com/. Accessed 16 January 2021

31. Tamura K, Mukai H, Naito Y, Yonemori K, Kodaira M, Tanabe Y, et al (2016) Phase I study of palbociclib, a cyclin-dependent kinase 4/6 inhibitor, in Japanese patients. Cancer Sci 107:755-763. http://doi:10.1111/cas.12932

32. Tchekmedyian V, Sherman EJ, Dunn L, Tran C, Baxi S, Katabi N, et al (2019) Phase II Study of Lenvatinib in Patients With Progressive, Recurrent or Metastatic Adenoid Cystic Carcinoma. J Clin Oncol 37:1529-1537. http://doi:10.1200/JC0.18.01859

33. Terhaard CH, Lubsen H, Van der Tweel I, Hilgers FJ, Eijkenboom WM, Marres HA, et al (2004) Salivary gland carcinoma: independent prognostic factors for locoregional control, distant metastases, and overall survival: results of the Dutch head and neck oncology cooperative group. Head Neck 26:681692. http://doi:10.1002/hed.10400

34. Thomson DJ, Silva P, Denton K, Bonington S, Mak SK, Swindell R, et al (2015) Phase II trial of sorafenib in advanced salivary adenoid cystic carcinoma of the head and neck. Head Neck 37:182187. http://doi:10.1002/hed.23577

35. van Tilburg CM, Milde T, Witt R, Ecker J, Hielscher T, Seitz A, et al (2019) Phase I/II intra-patient dose escalation study of vorinostat in children with relapsed solid tumor, lymphoma, or leukemia. Clin Epigenetics 11:188. http://doi:10.1186/s13148-019-0775-1

36. van Weert S, Bloemena E, van der Waal I, de Bree R, Rietveld DH, Kuik JD, Leemans CR (2013) Adenoid cystic carcinoma of the head and neck: a single-center analysis of 105 consecutive cases over a 30-year period. Oral Oncol 49:824-829. http://doi:10.1016/j.oraloncology.2013.05.004

37. Vorinostat. IBM Micromedex® DRUGDEX® (electronic version). IBM Watson Health, Greenwood Village, Colorado, USA. https://www.micromedexsolutions.com/. Accessed 16 January 2021

38. Wang D, Lippard SJ (2005) Cellular processing of platinum anticancer drugs. Nat Rev Drug Discov 4:307-320. http://doi:10.1038/nrd1691

39. Westerfield M (2000) Guide for the laboratory use of Zebrafish (Danio rerio): The Zebrafish Book. 4th ed. Eugene, University of Oregon Press

40. Xu B, Drill E, Ho A, Ho A, Dunn L, Prieto-Granada CN, Chan T, Ganly I, Ghossein R, Katabi N (2017) Predictors of Outcome in Adenoid Cystic Carcinoma of Salivary Glands: A Clinicopathologic Study With Correlation Between MYB Fusion and Protein Expression. Am J Surg Pathol 41:1422-1432. http://doi:10.1097/PAS.0000000000000918

41. Xu L, Li L, Zhang J, Cai W, Zhao S, Liu S (2020) Accumulated cytotoxicity of CDK inhibitor dinaciclib with first-line chemotherapy drugs in salivary adenoid cystic carcinoma cells. Odontology 108:300311. http://doi:10.1007/s10266-019-00451-5

42. Xu MJ, Wu TJ, van Zante A, El-Sayed IH, Algazi AP, Ryan WR, Ha PK, Yom SS (2018) Mortality risk after clinical management of recurrent and metastatic adenoid cystic carcinoma. J Otolaryngol Head Neck Surg 47:28. http://doi:10.1186/s40463-018-0273-z 
43. Younes MN, Park YW, Yazici YD, Gu M, Santillan AA, Nong X, Kim S, Jasser SA, El-Naggar AK, Myers JN (2006) Concomitant inhibition of epidermal growth factor and vascular endothelial growth factor receptor tyrosine kinases reduces growth and metastasis of human salivary adenoid cystic carcinoma in an orthotopic nude mouse model. Mol Cancer Ther 5:2696-2705. http://doi:10.1158/1535-7163.MCT-05-0228

44. Zhang B, Xuan C, Ji Y, Zhang W, Wang D (2015) Zebrafish xenotransplantation as a tool for in vivo cancer study. Fam Cancer 14:487-493. http://doi:10.1007/s10689-015-9802-3

45. Zhu G, Chang P, Lippard SJ (2010) Recognition of platinum-DNA damage by poly(ADP-ribose) polymerase-1. Biochemistry 49:6177-6183. http://doi:10.1021/bi100775t

\section{Tables}

Table 1 Drugs potency and efficacy

\begin{tabular}{|lll|}
\hline Drugs & IC $_{50}$ (95\% C.I.) & \% cell mortality ( \pm SEM) at max concentration (M) \\
\hline cisplatin & $3 \mu \mathrm{M}(2.3$ to $3.5 \mu \mathrm{M})$ & $91.15 \%( \pm 2.6)$ at $24 \mu \mathrm{M}$ \\
\hline doxorubicin & $0.013 \mu \mathrm{M}(0.01$ to $0.017 \mu \mathrm{M})$ & $94.91 \%( \pm 0.77)$ at $1 \mu \mathrm{M}$ \\
\hline vorinostat & $1.3 \mu \mathrm{M}(1.1$ to $1.6 \mu \mathrm{M})$ & $96.28 \%( \pm 0.47)$ at $13.5 \mu \mathrm{M}$ \\
\hline olaparib & $5.5 \mu \mathrm{M}(3.8$ to $8.1 \mu \mathrm{M})$ & $74.26 \%( \pm 2.69)$ at $18 \mu \mathrm{M}$ \\
\hline palbociclib & $0.13 \mu \mathrm{M}(0.09$ to $0.2 \mu \mathrm{M})$ & $60.13 \%( \pm 3.4)$ at $9 \mu \mathrm{M}$ \\
\hline everolimus & $0.5 \mathrm{nM}(0.3$ to $0.8 \mathrm{nM})$ & $48.96 \%( \pm 2.23)$ at $250 \mathrm{nM}$ \\
\hline
\end{tabular}

\section{Figures}



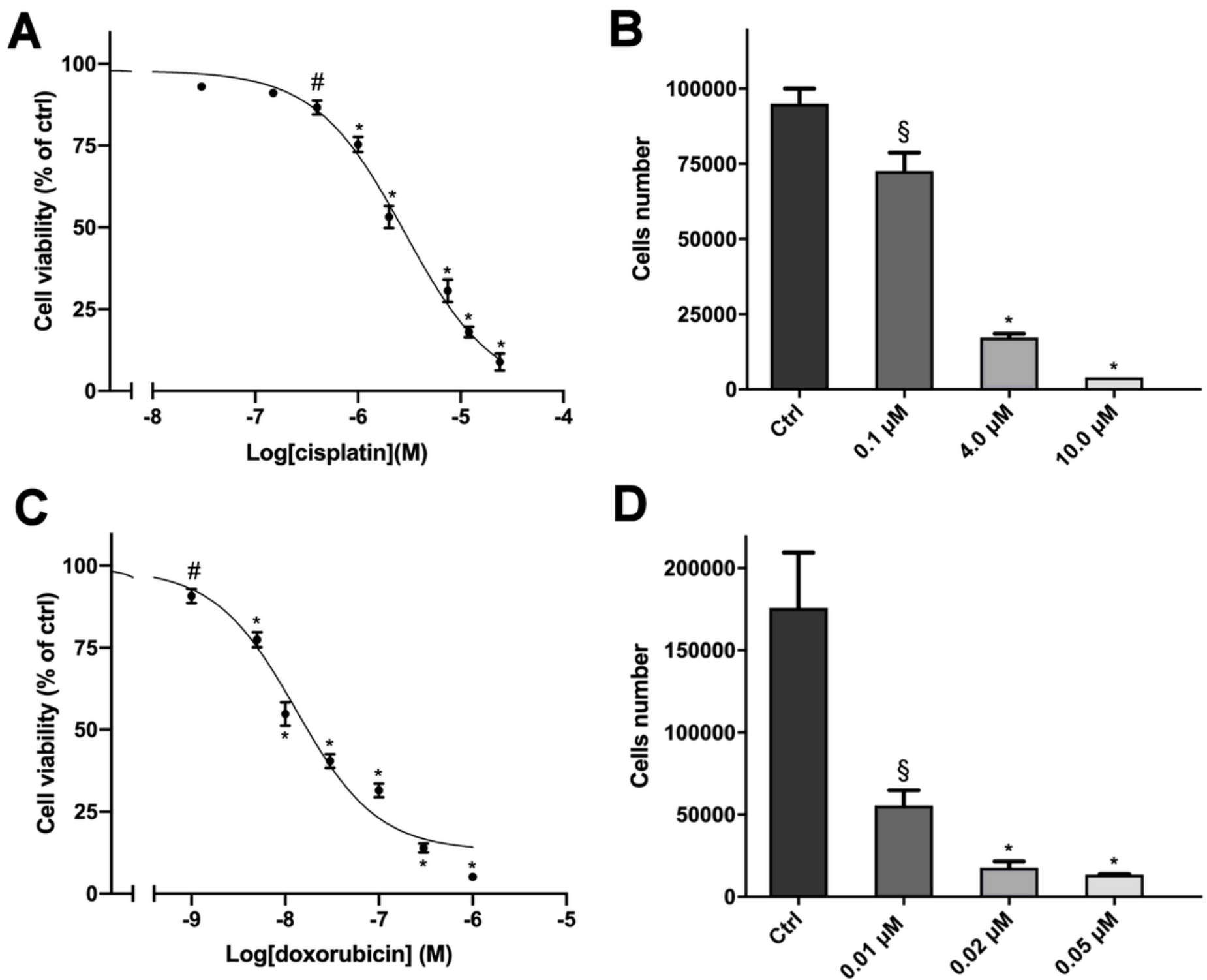

\section{Figure 1}

Cytotoxic effect of cisplatin and doxorubicin in hTERT cells. (A) Concentration-response curve of cisplatin-induced inhibition of cell viability. Cells were treated with increasing concentrations of cisplatin (0.03-24 $\mu \mathrm{M})$ for 4 days. (B) Effect of cisplatin on cell proliferation. Cells were treated for 4 days with three concentrations of cisplatin. (C) Concentration-response curve of doxorubicin-induced inhibition of cell viability. Cells were treated with increasing concentrations of doxorubicin $(0.001-1 \mu \mathrm{M})$ for 4 days. (D) Effect of doxorubicin on cell proliferation. Cells were treated for 4 days with three concentrations of doxorubicin. Cell viability was evaluated by MTT assay while cells proliferation was assessed after cells count with trypan blue exclusion. Data are shown as mean \pm SEM. $\left[{ }^{\star} p<0.0001 ; \# p<0.01 ; \S p<0.05\right]$ 

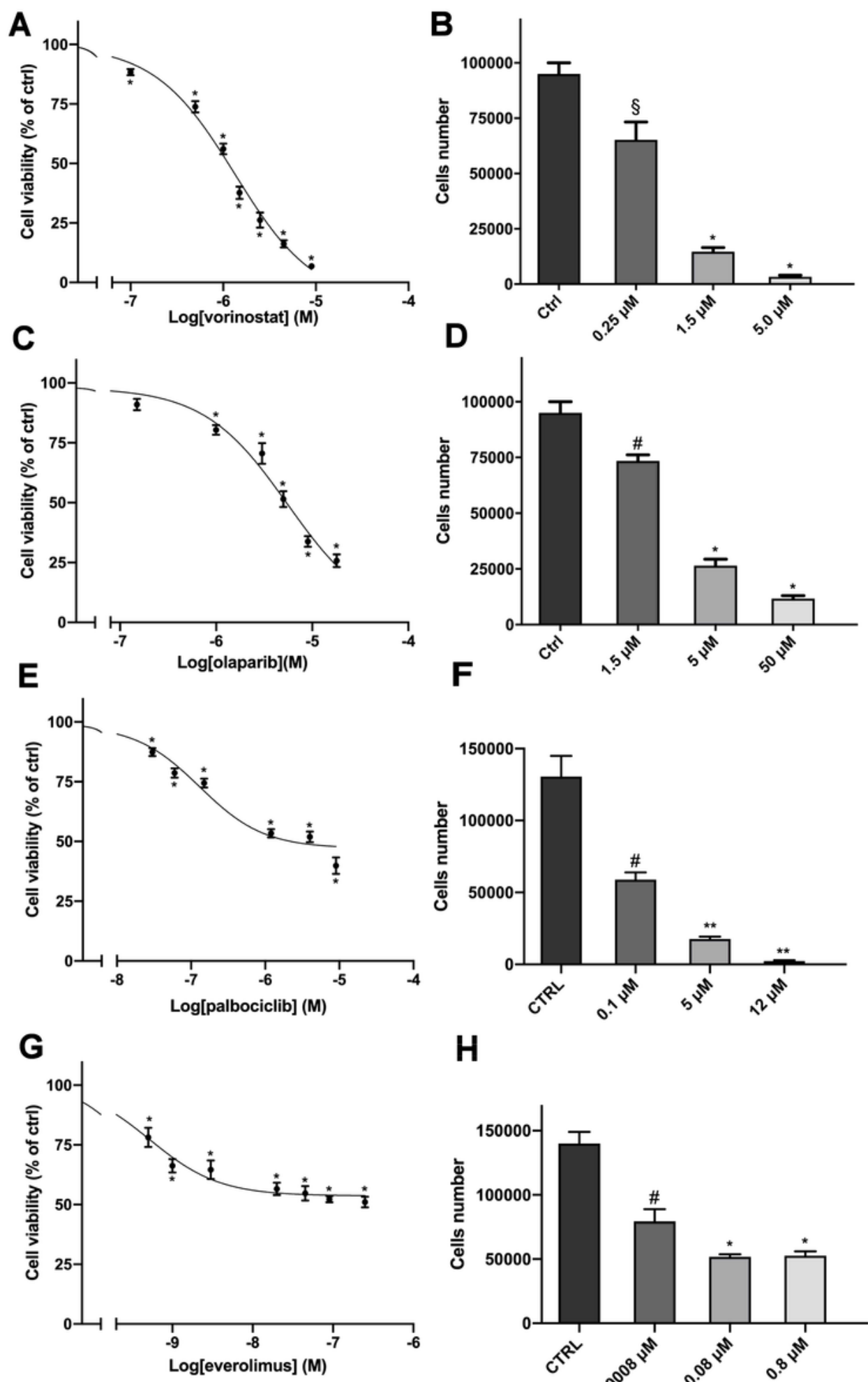

H

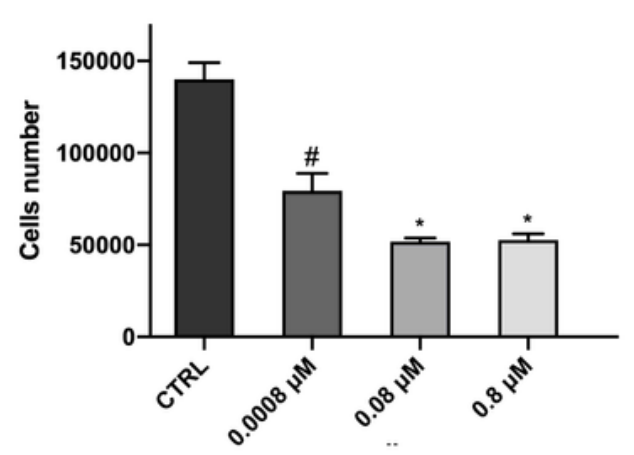

Figure 2

Cytotoxic effect of target therapy drugs in hTERT cells. (A, C, E, G) Concentration-response curves of vorinostat-, olaparib-, palbociclib- and everolimus-induced inhibition of cell viability. Cells were treated with increasing concentrations of vorinostat $(0.1-9 \mu \mathrm{M})$ or olaparib $(0.15-18 \mu \mathrm{M})$ or palbociclib (0.03-9 $\mu \mathrm{M})$ or everolimus (0.5-250 nM) for 4 days. (B, D, F, H) Effect of vorinostat, olaparib, palbociclib and everolimus respectively, on cell proliferation. Cells were treated for 4 days with three concentrations of 
each drug. Cell viability was evaluated by MTT assay while cells proliferation was assessed after cells count with trypan blue exclusion. Data are shown as mean \pm SEM. $\left[{ }^{*} p<0.0001 ; \# p<0.01 ; \S p<0.05\right]$
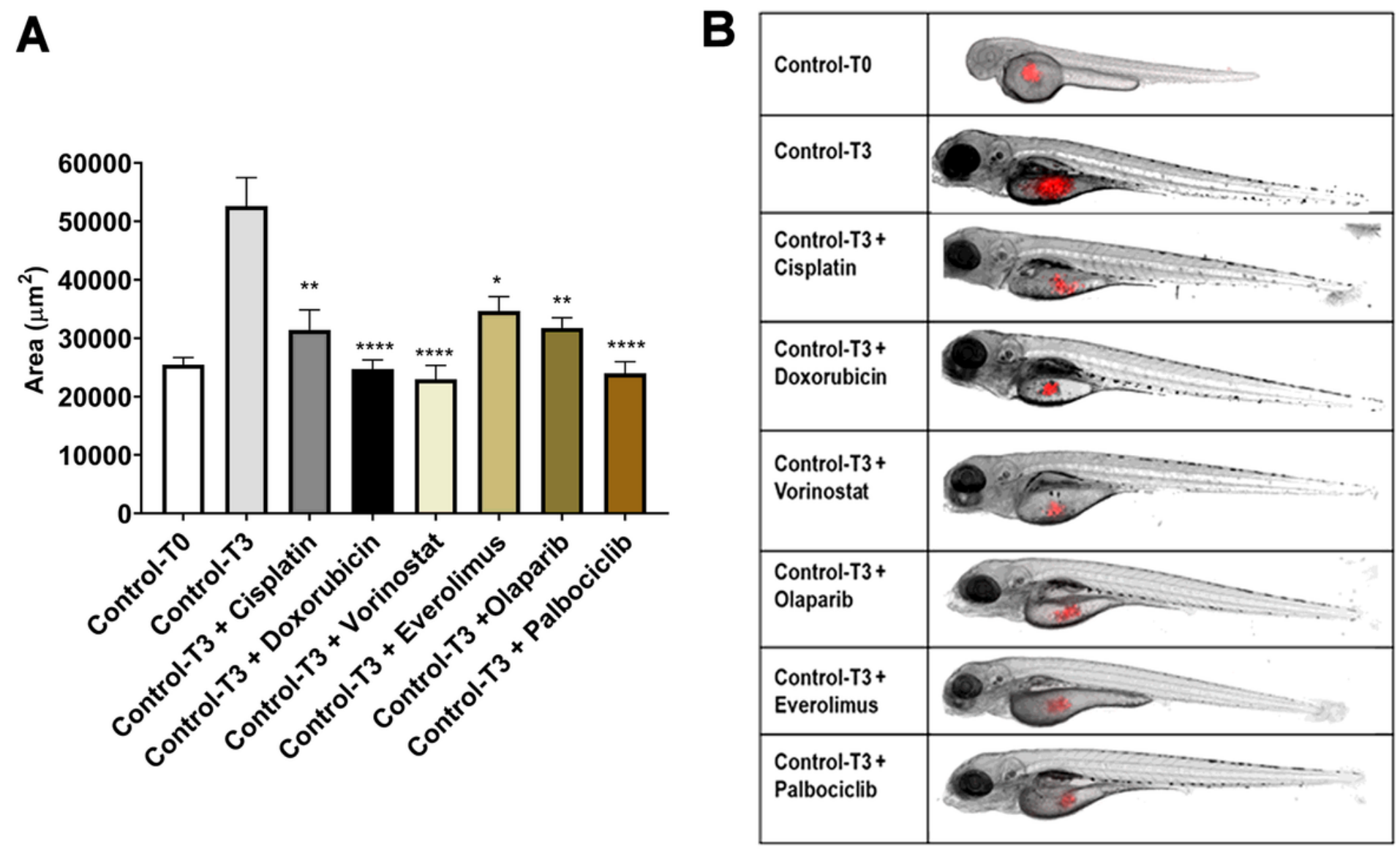

\section{Figure 3}

Chemotherapy drugs and target therapy drugs reduced the tumor xenograft area of hTERT cells. (A) The tumor area of T0 and T3 drug-treated and solvent-treated groups was measured with Noldus DanioScopeTM software. (B) A representative image is shown. Control-T0, time point at injection (control embryos at $48 \mathrm{hpf}$ ); Control-T3, time point 3 days later in fish water with solvent alone (untreated embryos at $120 \mathrm{hpf}$ ); Control-T3+drugs, time point 3 days later in fish water with drugs (treated embryos at $120 \mathrm{hpf}$ ). The experiments were performed twice and each group was representative of 25 embryos.

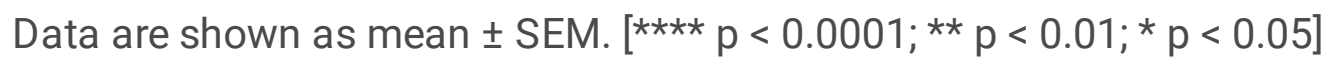



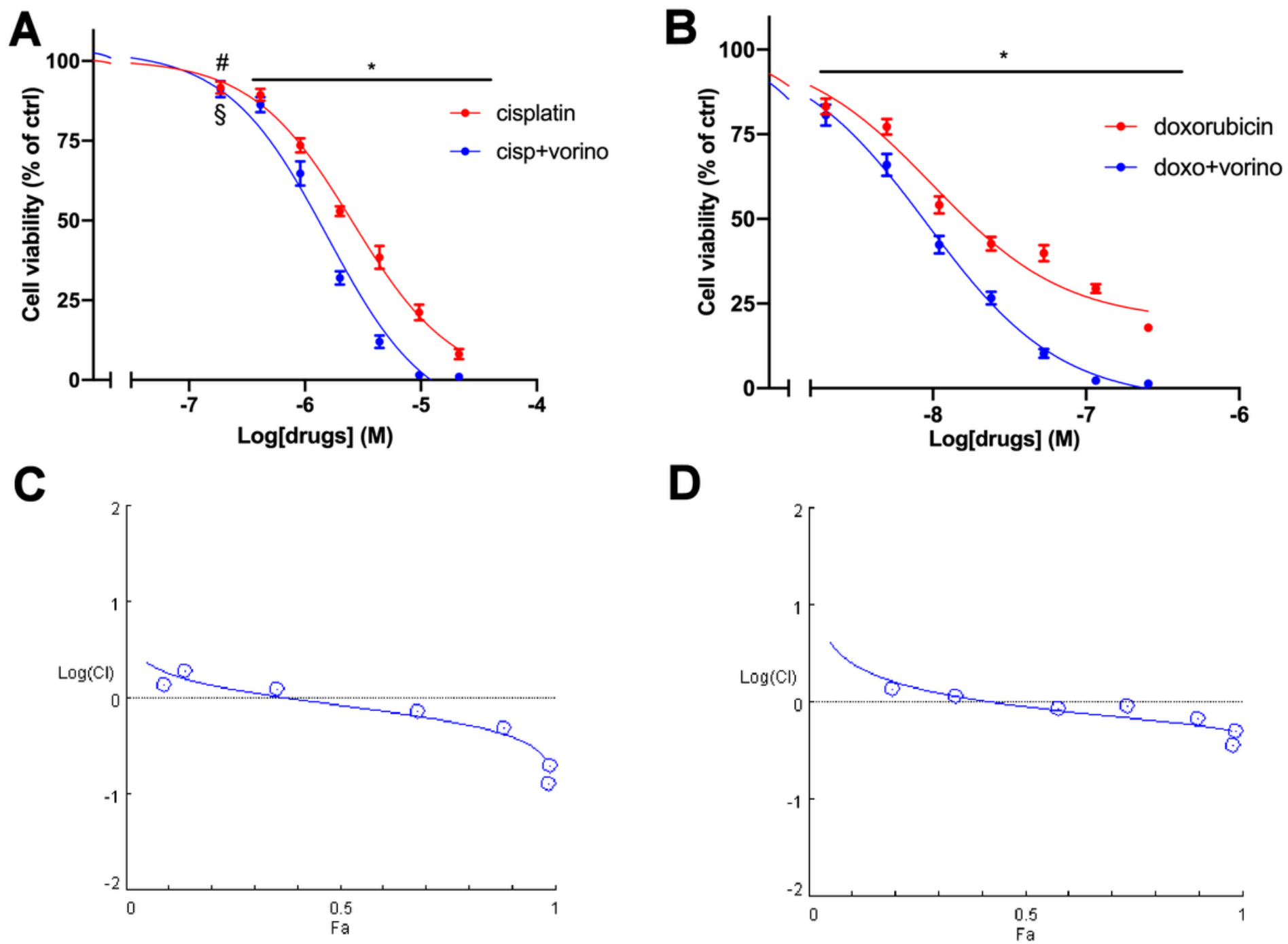

D

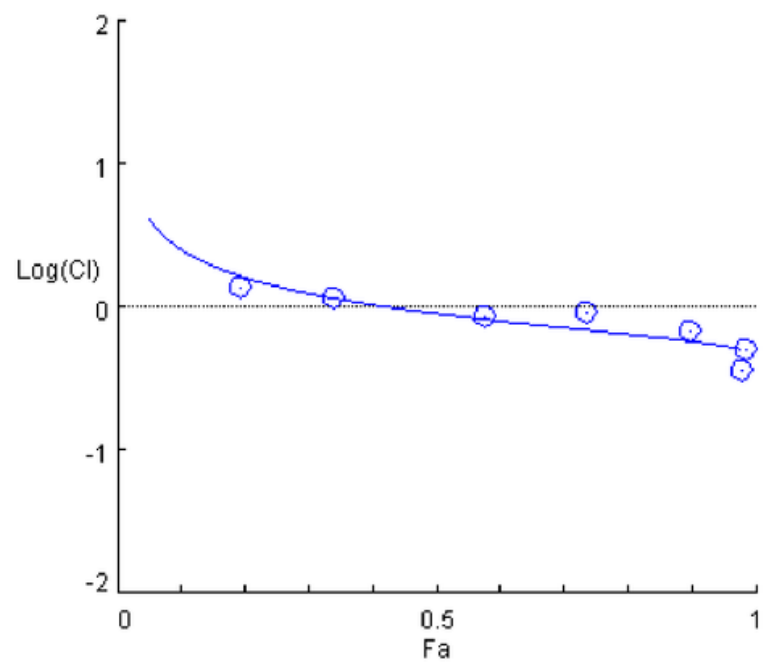

\section{Figure 4}

Vorinostat enhanced the effect of the chemotherapy drugs on hTERT cell viability. (A) Concentrationresponse curves of cisplatin- and cisplatin plus vorinostat-induced inhibition of cell viability. Cells were treated with increasing concentrations of cisplatin and vorinostat alone or in combination at fixed concentration molar ratio (cisplatin : vorinostat $=2.9: 1$ ) for 4 days. (B) Concentration-response curves of doxorubicin- and doxorubicin plus vorinostat-induced inhibition of cell viability. Cells were treated with increasing concentrations of doxorubicin and vorinostat alone or in combination at fixed concentration molar ratio (doxorubicin : vorinostat $=1: 62.7$ ) for 4 days. Cell viability was evaluated by MTT assay. Data are shown as mean \pm SEM. (C, D) Semilogarithmic-Combination Index Plot of combined treatments with cisplatin plus vorinostat and doxorubicin plus vorinostat respectively. Dose and effect data obtained were converted to Fa values and analyzed with CompuSyn software [ ${ }^{*} p<0.0001 ; \# p<0.01 ; \S p<0.05$ ] 

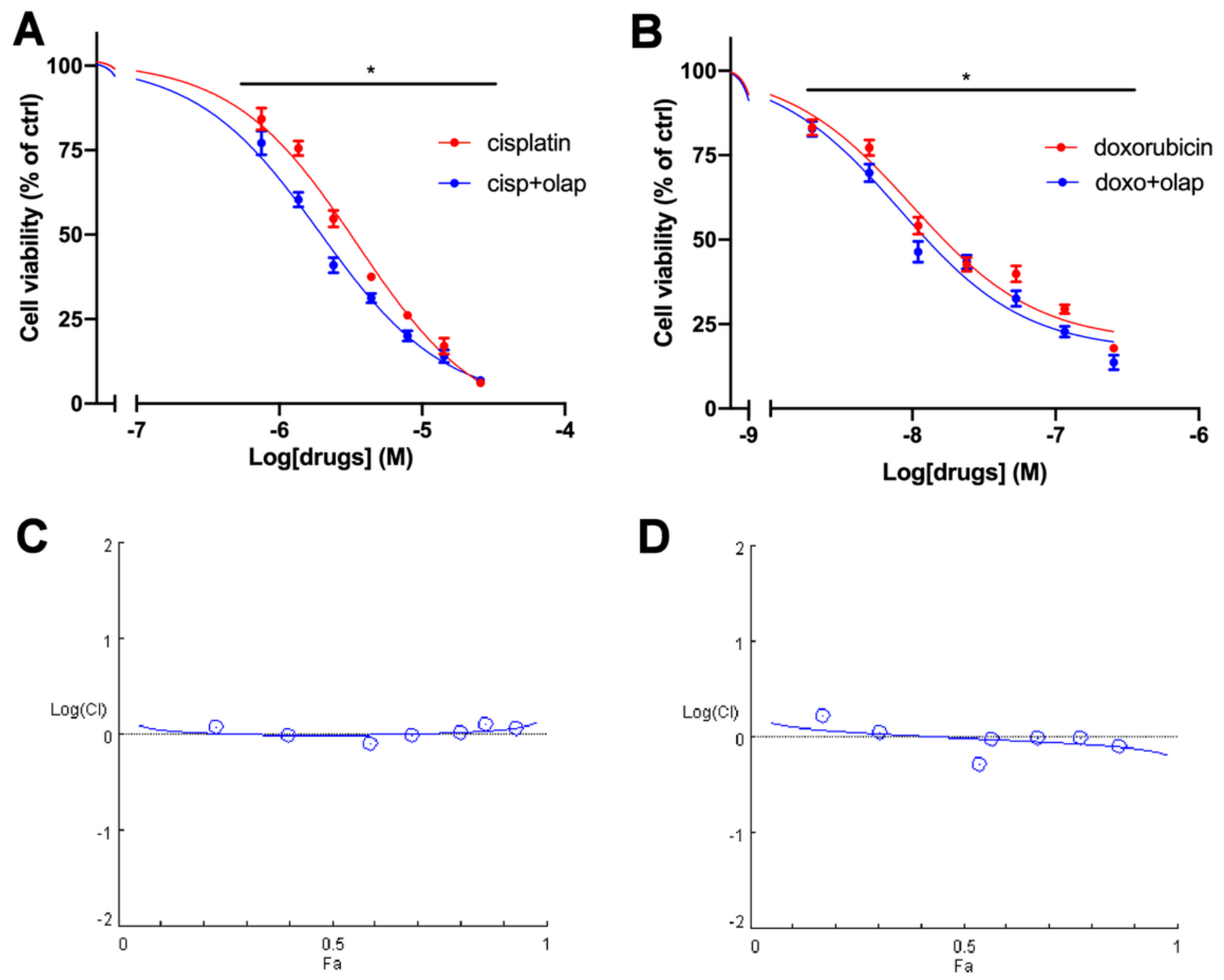

\section{Figure 5}

Olaparib enhanced the effect of the chemotherapy drugs on hTERT cell viability. (A) Concentrationresponse curves of cisplatin- and cisplatin plus olaparib-induced inhibition of cell viability. Cells were treated with increasing concentrations of cisplatin and olaparib alone or in combination at fixed concentration molar ratio (cisplatin : olaparib $=1: 1.2$ ) for 4 days. (B) Concentration-response curves of doxorubicin- and doxorubicin plus olaparib-induced inhibition of cell viability. Cells were treated with increasing concentrations of doxorubicin and olaparib alone or in combination at fixed concentration molar ratio (doxorubicin : olaparib $=1: 104.3$ ) for 4 days. Cell viability was evaluated by MTT assay. Data are shown as mean \pm SEM. $(C, D)$ Semilogarithmic-Combination Index Plot of combined treatments with cisplatin plus olaparib and doxorubicin plus olaparib respectively. Dose and effect data obtained were converted to Fa values and analyzed with CompuSyn software $\left[{ }^{*} p<0.0001\right]$ 

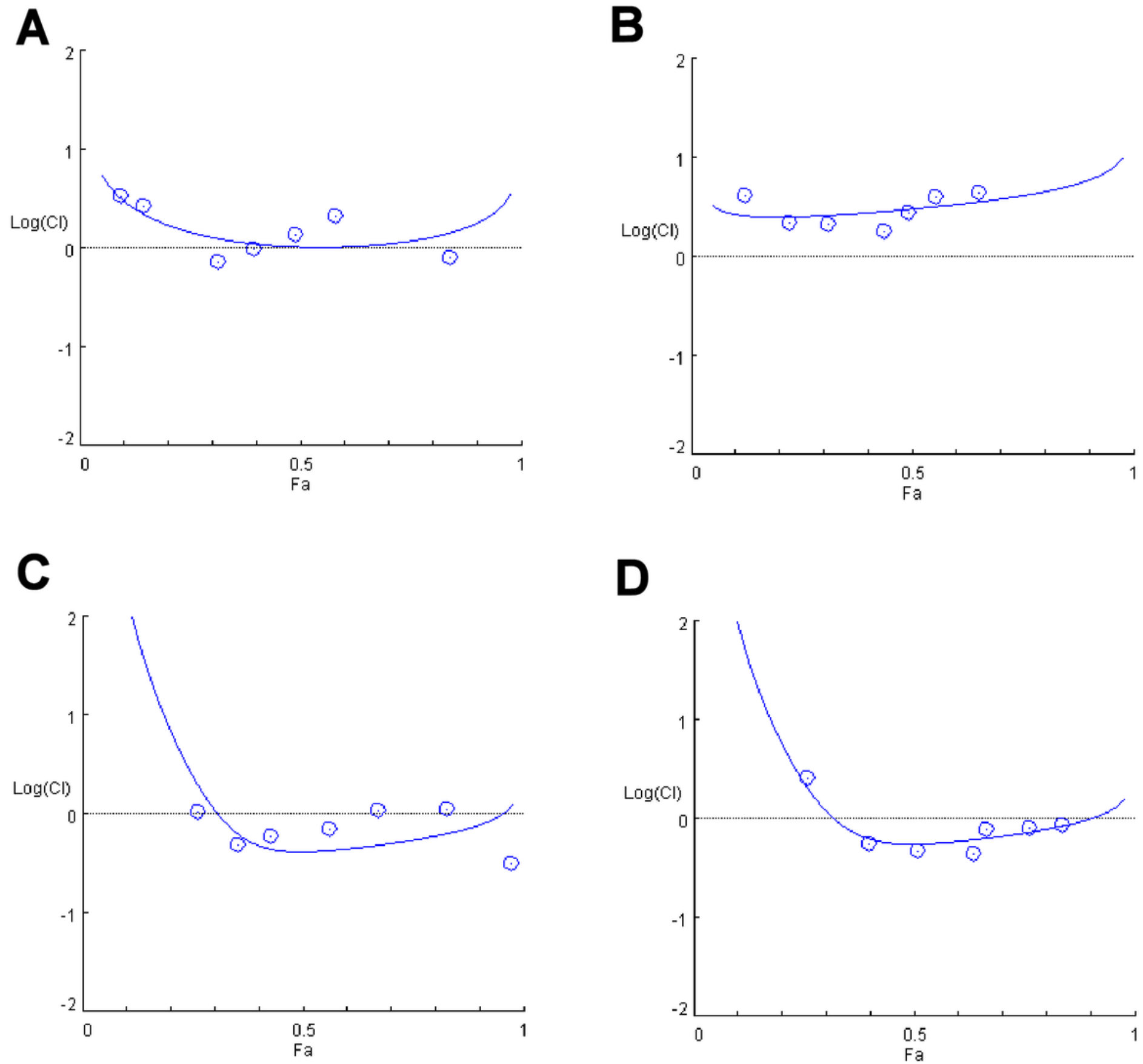

\section{D}

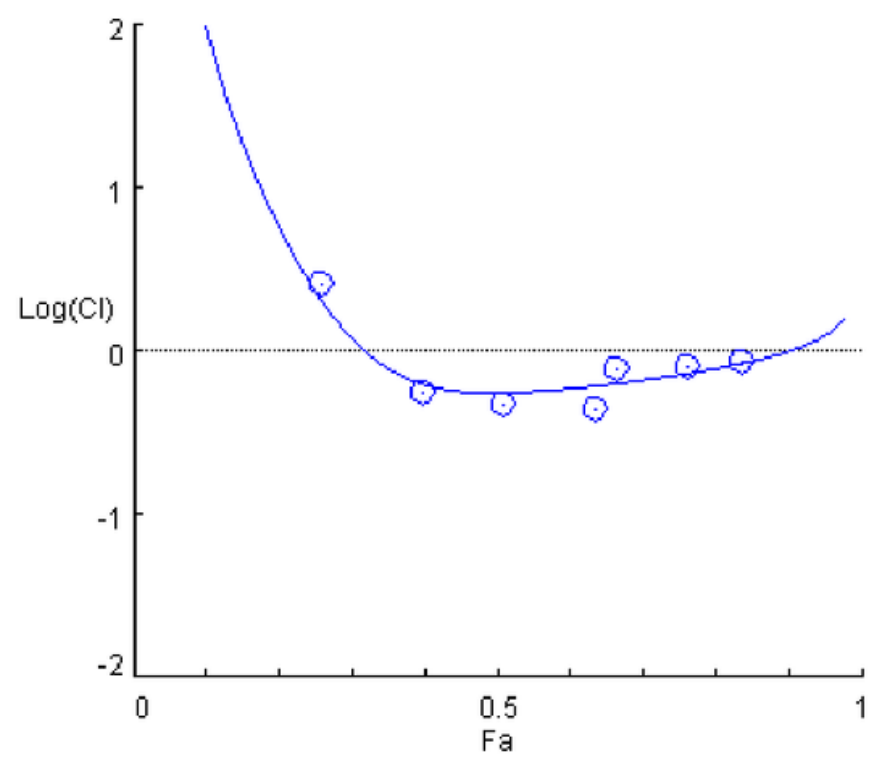

\section{Figure 6}

Effect of palbociclib and everolimus combined with chemotherapy drug. Semilogarithmic-Combination Index Plot of combined treatments with (A) cisplatin plus palbociclib, (B) doxorubicin plus palbociclib, (C) cisplatin plus everolimus and (D) doxorubicin plus everolimus. Dose and effect data obtained were converted to Fa values and analyzed with CompuSyn software 